\title{
Quantitative Analysis of Bilobalide and Ginkgolides from Ginkgo biloba Leaves and Ginkgo Products Using ${ }^{1} \mathrm{H}-\mathrm{NMR}$
}

\author{
Young Hae Chol, ${ }^{a}$ Hyung-Kyoon Chor,${ }^{a}$ Arno HazeKamp, ${ }^{a}$ Paloma Bermejo, ${ }^{a}$ Yvonne Schilder, ${ }^{a}$ \\ Cornelis ERKELENS, ${ }^{b}$ and Robert VerPOORTE* ${ }^{*}, a$ \\ ${ }^{a}$ Division of Pharmacognosy, Leiden/Amsterdam Center for Drug Research, Gorlaeus Laboratories, Leiden University; \\ P. O. Box 9502, 2300 RA Leiden, The Netherlands: and ${ }^{b}$ Division of NMR, Leiden Institute of Chemistry, Gorlaeus \\ Laboratories; P. O. Box 9502, 2300 RA Leiden, The Netherlands.
}

Received September 17, 2002; accepted November 19, 2002

\begin{abstract}
${ }^{1} \mathrm{H}-\mathrm{NMR}$ spectrometry was applied to the quantitative analysis of the bilobalide, ginkgolides $\mathrm{A}, \mathrm{B}$, and $\mathrm{C}$ in Ginkgo biloba leaves and six kinds of commercial Ginkgo products without any chromatographic purification. The experiment was performed by the analysis of each singlet $\mathrm{H}-12$, which were well separated in the range of $\delta$ 6.0-7.0 in the ${ }^{1} \mathrm{H}-\mathrm{NMR}$ spectrum. However, the $\mathrm{H}-12$ protons of bilobalide and ginkgolides may have overlapped with H-6 or H-8 protons of the Ginkgo flavonoids. Therefore, the optimum ${ }^{1} \mathrm{H}-\mathrm{NMR}$ solvent for the analysis of the compound was selected through the evaluation of solvent effects on the resolution of these signals from the compounds. Acetone- $d_{6}$-benzene- $d_{6}(50: 50)$ was found to be the best one among the solvents evaluated. The quantity of the compounds was calculated by the relative ratio of the intensity of each compound to the known amount of internal standard $(25 \mu \mathrm{g} / \mathrm{ml})$, phloroglucinol. This method allows rapid and simple quantitation of underivatized bilobalide and ginkgolides in $5 \mathrm{~min}$ without any pre-purification steps.
\end{abstract}

Key words bilobalide; ginkgolide; ${ }^{1} \mathrm{H}-\mathrm{NMR}$ spectrometry; quantitative analysis; Ginkgo biloba leaves; Ginkgo products

The Ginkgo tree has been of interest to mankind for more than 2000 years, making it one of the oldest known medicinal plants. It is of great interest because its leaves possess pharmacological properties that include radical scavenging, improved blood flow, vasoprotection, and anti-PAF (platelet aggregating factor) activity. ${ }^{1,2}$ In numerous experimental models, a ginkgo extract was found to affect vascular and metabolic disturbances as well as neurological and behavioral activities. ${ }^{3-6)}$ Among the constituents of Ginkgo biloba, terpene trilactones such as bilobalide (BB), ginkgolides $\mathrm{A}$ (GA), B (GB), and C (GC) (Fig. 1), along with flavonoids, have been identified as the active constituents of the Ginkgo extract for inhibition of the binding of $\mathrm{PAF}^{7,8)}$ Recently, these compounds have also been reported to be effective in the treatment of cerebrovascular insufficiency and peripheral circulatory problems, as well as Alzheimer's disease. ${ }^{9-12)}$

For the analysis of these bioactive constituents, HPLC with UV detection has been developed but many trace impurities still interfere because of very poor UV absorption characteristics of the ginkgolides. ${ }^{13,14)}$ Therefore, HPLC with RI or ELSD, and GC with FID or MS are more suitable and have been used with considerable success. However, low sensitivity, baseline instability, and need of extensive clean up steps still remain a problem. ${ }^{15-17)}$

To solve the analytical problems of these Ginkgo terpene trilactones, the development of a reliable and robust method is required as alternative to the conventional analyses. Previously, a quantitative method using ${ }^{1} \mathrm{H}-\mathrm{NMR}$ spectrometer was applied for the analysis of these compounds after a chromatographic preparation step. ${ }^{18)}$ This method, however, requires a chromatographic separation because some impurities could interfere with the NMR analysis. The impurities may be mainly the Ginkgo flavonoids such as isorhamnetin, kaempferol, myricetin, quercetin, or their analogues. In particular, the signals of H-6 and H-8 of the flavonoids may interfere with the target proton signals of bilobalide and ginkgolides because the protons of the flavonoids were de- tected in a relatively upfield region compared to other phenyl protons. ${ }^{19)}$ Therefore in this study, we intended to develop an alternative analytical method using ${ }^{1} \mathrm{H}-\mathrm{NMR}$ for Ginkgo terpene lactones without any tedious chromatographic purification. The developed method was applied on the quantitative analysis of Ginkgo leaves and six kinds of commercial Ginkgo products for bilobalide, ginkgolides A, B, and C.

\section{Experimental}

Solvents and Chemicals First grade methanol, cyclohexane, and methylethylketone were purchased from Merck Biosolve Ltd. Valkenswaard, the Netherlands and $\mathrm{Na}_{2} \mathrm{SO}_{4}$ from Merck (Darmstadt, Germany). Acetone- $d_{6}$ $(99.96 \%)$ and benzene- $d_{6}(99.96 \%)$ were purchased from Cortec (Paris, France). Phlororoglucinol (1,3,5-trihydroxybenzene) from Sigma (St. Louis,

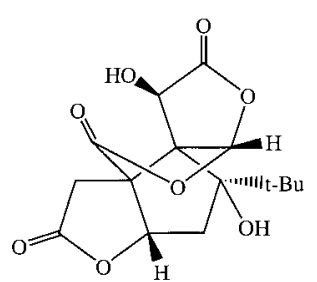

Bilobalide (BB)

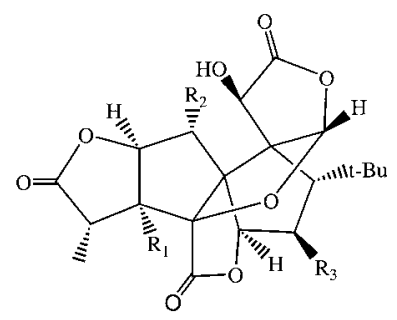

Ginkgolide $A(G A): R_{1}=O H, R_{2}=R_{3}=H$ Ginkgolide $B(G B): R_{1}=R_{2}=O H, R_{3}=H$ Ginkgolide $C(\mathrm{GC}): \mathrm{R}_{1}=\mathrm{R}_{2}=\mathrm{R}_{3}=\mathrm{OH}$<smiles>O=c1c(O)c(-c2cc([18OH])c(O)c([123I])c2)oc2cc(O)cc(O)c12</smiles>

Fig. 1. Chemical Structures of Bilobalide (BB), Ginkgolide A (GA), B (GB), C (GC), Isorhamnetin, Kaempferol, Myricetin, and Quercetin 
MO, U.S.A.), kaempferol from Fluka, Buchs, Switzerland, myricetin (Fluka, Buchs, Swiss), quercetin (Fluka, Buchs, Switzerland), quercitrin (Fluka, Buchs, Switzerland), and rutin (Labaz, Brussel, Belgium) were used as reference compounds. Bilobalide, ginkolides $\mathrm{A}, \mathrm{B}$, and $\mathrm{C}$ were generously donated by GreenTek21 (Seoul, Korea).

${ }^{1}$ H-NMR Apparatus and Parameters ${ }^{1} \mathrm{H}-\mathrm{NMR}$ spectra were recorded in acetone- $d_{6}$, benzene- $d_{6}$, and the mixture of them $(25: 75,50: 50$, and $75: 25$, respectively) using a Bruker DMX 600 spectrometer (equipped with an Indy Silicon graphics computer). For each sample, 64 scans were recorded with the following parameters: $0.167 \mathrm{~Hz} /$ point, pulse width $(\mathrm{PW})=$ $4.0 \mu \mathrm{s}$, and relaxation delay $(\mathrm{RD})=1.0 \mathrm{~s}$. FIDs were Fourier transformed with $\mathrm{LB}=0.2 \mathrm{~Hz}, \mathrm{~GB}=0$, and $\mathrm{PC}=1.0$. For quantitative analysis, peak height was used.

Evaluation of Recovery of Bilobalide, Ginkgolides A, B, and C One gram of filter paper disks $\left(589^{2}\right.$ white ribbon ashless, Schleicher \& Schuell, $\mathrm{GmbH}$, Cassel, Germnay) was cut into $c a .1 \mathrm{~cm}$ diameter and placed in the extraction vessel. Each standard of bilobalide, ginkgolides A, B, C, myricetin, kaempferol, quercetin, quercitrin, and rutin $(1.0 \mathrm{mg})$ was spiked into the filter paper disks. Then the spiked samples were dried in vacuum oven at $40^{\circ} \mathrm{C}$ for $24 \mathrm{~h}$.

Plant Material and Commercial Ginkgo Product To analyse samples of wide variety, leaves (Ginkgo biloba L.) were collected in Seoul, Korea in May 2002 and Essential Organics ${ }^{\circledR}$ (Bio San Labs., Inc., Derry, NH, U.S.A.), Lucovit $^{\mathbb{R}}$ (PK Benelux, Uden, the Netherlands), Bio-Biloba ${ }^{\circledR}$ (Pharma Nord, Vejle, Denmark), Vitelle ${ }^{\circledR}$ (Indros B.V., Almere, the Netherlands), Geriaforce $^{\mathbb{B}}$ (Biohorma, B.V., Elburg, the Netherlands), and Ginkgo Biloba $\phi^{\circledR}$ (VSM Geneesmiddelen B.V., Alkmaar, the Netherlands), all purchased in a pharmacy, were used for the analysis.

Extraction The extraction method was performed according to Komoda's method. ${ }^{20)}$ Five hundred milligrams of a sample in $100 \mathrm{ml}$ of $70 \%$ $\mathrm{MeOH}$ was ultrasonicated for $30 \mathrm{~min}$ with three times. The three extracts were combined and evaporated to dryness using a rotary evaporator. The $70 \% \mathrm{MeOH}$ extracts were partitioned between cyclohexane $(30 \mathrm{ml} \times 3$ times $)$ and water $(30 \mathrm{ml})$. The water fractions were re-partitioned by methylethylketone ( $30 \mathrm{ml} \times 3$ times). $\mathrm{Na}_{2} \mathrm{SO}_{4}$ was used for removing remaining water from the methylethylketone. The methylethylketone fractions were evaporated and reconstituted in $1 \mathrm{ml}$ of the mixture of acetone- $d_{6}$ and benzene- $d_{6}(1: 1)$. All experiments were based on triplicate.

\section{Results and Discussion}

To achieve our goal, the quantitation of bilobalide (BB), ginkgolide A (GA), B (GB), and C (GC) in Ginkgo leaves and commercial pharmaceutical preparations by using ${ }^{1} \mathrm{H}$ NMR, it should be possible to quantify each individual ginkgolide by means of the integral or intensity of a specific proton signal. In a previous report, the H-12 protons of bilobalide and ginkgolides could be well separated and appeared as singlets. ${ }^{18)}$ However, the ${ }^{1} \mathrm{H}-\mathrm{NMR}$ peaks of Ginkgo flavonoids such as isorhamnetin, kaempferol, myricetin, quercetin, or their derivatives may interfere with the target $\mathrm{H}$ 12 peaks of the Ginkgo terpenes. Therefore, some tedious cleaning or preparation step might be required for the analysis. In particular, H-6 or H-8 of each flavonoid can overlap with the peaks of the Ginkgo terpenes in the ${ }^{1} \mathrm{H}-\mathrm{NMR}$ spectra since these peaks are shifted to the upfield region by the adjacent 5-OH and 7-OH. To solve this problem, changing NMR solvent, controlling $\mathrm{pH}$, and an addition of an NMR shifting reagent were evaluated. Of these, the addition of benzene- $d_{6}$ into acetone- $d_{6}$, was found to shift the ${ }^{1} \mathrm{H}-\mathrm{NMR}$ peaks of the H- 6 or H-8 of the flavonoids to lower field and thus to be the optimum NMR solvent for the Ginkgo terpenes analysis. This NMR solvent showed a good separation of the H-12 peaks of bilobalide, ginkgolide A, B, and C. Figure 2 shows the effect of the percentage of benzene- $d_{6}$ on the separation of these signals. The mixture of benzene- $d_{6}$ and acetone- $d_{6}(50: 50)$ gave a good separation of all peaks originating from flavonoids and terpenoids. As an appropriate in-

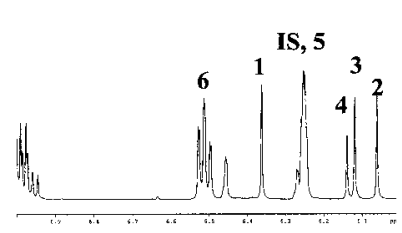

$\mathbf{A}$

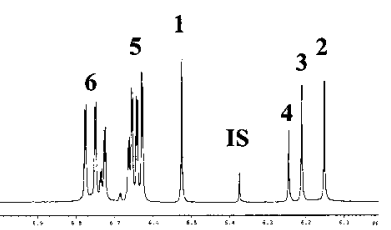

C

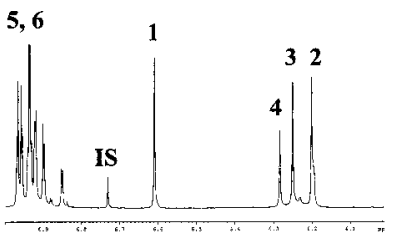

B

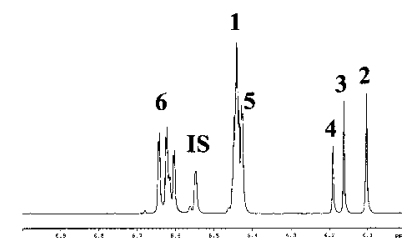

D
Fig. 2. $600 \mathrm{MHz}{ }^{1} \mathrm{H}-\mathrm{NMR}$ Spectrum of the Reference Compounds in Acetone- $d_{6}$ (A), Acetone- $d_{6}-$ Benzene- $d_{6}(75: 25)$ (B), Acetone- $d_{6}-$ Benzene- $d_{6}$ $(50: 50)(\mathrm{C})$, and Acetone- $d_{6}-$ Benzene- $d_{6}(25: 75)$ (D) in the Range of $\delta$ $6.0-7.0$

1: $\mathrm{H}-12$ of bilobalide (BB), 2: H-12 of ginkgolide A (GA), 3: H-12 of ginkgolide B (GB), 4: H-12 of ginkgolide C (GC), 5: H-6 of reference flavonoids, 6: H-8 of reference flavonoids, IS: internal standard (phloroglucinol).

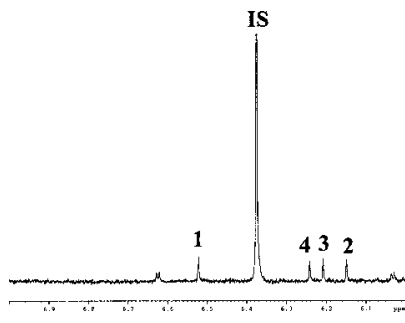

A

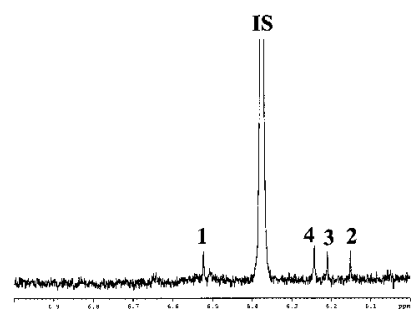

Fig. 3. $\quad 600 \mathrm{MHz}{ }^{1} \mathrm{H}-\mathrm{NMR}$ Spectrum of $10 \mathrm{ppm}$ (A) Using 64 Scans and $1 \mathrm{ppm}$ Using 1024 Scans (B) of the Reference Compounds in Acetone$d_{6}$-Benzene- $d_{6}(50: 50)$ in the Range of $\delta 6.0-7.0$

1: H-12 of bilobalide (BB), 2: H-12 of ginkgolide A (GA), 3: H-12 of ginkgolide B (GB), 4: H-12 of ginkgolide C (GC), IS: internal standard (phloroglucinol).

ternal standard, phloroglucinol (1,3,5-trihydroxybenzene) was used because it is stable, nonvolatile, and has a sharp singlet in the same areas of the target compounds but well separated. In the case of ginkgolic acids, other major phenolic constituents of Ginkgo biloba, the phenolic peaks are fortunately shown in a more downfield region (above $\delta$ 6.8) when compared to bilobalide and ginkgolides, and could be largely removed by the cyclohexane fractionation used in this study.

As the next step, the detection limit was measured for 64 scans of an extract of $500 \mathrm{mg}$ plant material (acquisition time: $4 \mathrm{~min}$ ). In this case, $10 \mathrm{ppm}$ of each compound was fully detected. Even $1 \mathrm{ppm}$ could be detected by increasing to 1024 scans (Fig. 3). The method can also be used to determine the absolute purity of reference substances for HPLC or GC determinations which is difficult to accomplish by other means as $100 \%$ pure standards of terpene trilactones are currently not available. The calibration curves for each compound using the ratio of the height of the compound and the internal standard were determined in the range of $0.01-$ 


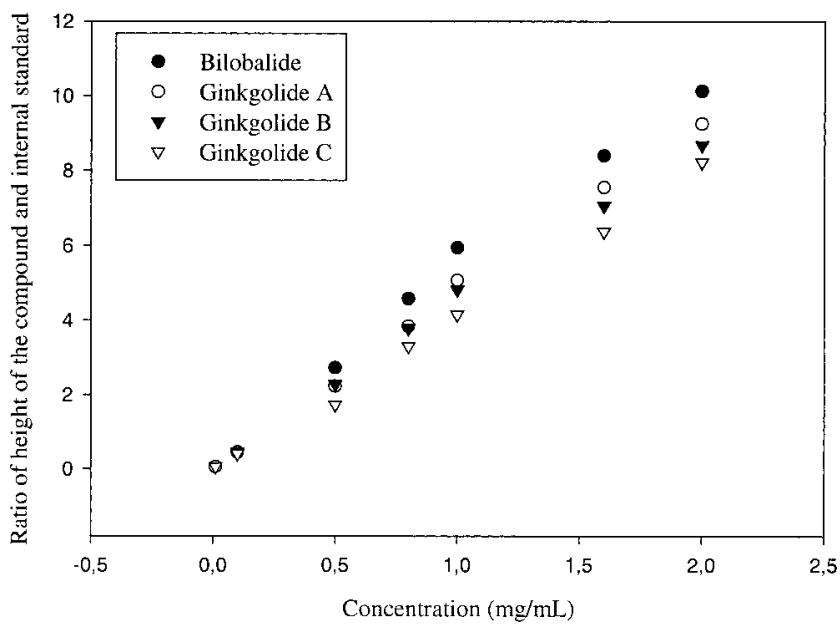

Fig. 4. Plot of the Area of H-12 Peaks of Bilobalide, Ginkgolide A, B, and $\mathrm{C}$ versus the Amounts of Each Compound

$2.0 \mathrm{mg} / \mathrm{ml}$ in order to evaluate the accuracy of this method depending on the different concentrations. Each calibration curve is shown in Fig. 4. The linearity of bilobalide (BB), ginkgolide A (GA), ginkgolide $\mathrm{B}$ (GB), and ginkgolide C (GC) were found to be larger than 0.990 , that is, 0.9925 , 0.9976, 0.9969, and 0.9978, respectively. However, we do not need these calibration curves for quantitation of the compounds because the integration or intensity of the peaks is always proportional to the amount of the compound and the same for all compounds.

For testing the recovering from a matrix, $1.0 \mathrm{mg}$ of each compound was extracted from cellulose papers on which the compounds were adsorbed. The extraction method consists of the simplest extraction and liquid-liquid fractionation scheme among previous reported sample preparation methods for Ginkgo terpene trilactones as we wanted to avoid some tedious clean-up steps using column chromatography. The recovery of each Ginkgo terpene trilactone with the chosen method was more than $96.0 \%$ (Table 1).

Finally, Ginkgo biloba leaves and six kinds of Ginkgo products were analyzed for bilobalide (BB), ginkgolide $\mathrm{A}$ (GA), B (GB), and C (GC) using the ${ }^{1} \mathrm{H}-\mathrm{NMR}$ method evaluated in this paper. For all samples evaluated in this study, the H-12 peak of each Ginkgo terpenes was quite well separated from the others and no interference was observed in the range of $\delta 6.0-7.0$ in the ${ }^{1} \mathrm{H}-\mathrm{NMR}$ spectrum (Fig. 5). In particular, sample 2 contained additional plant materials such as Rutae herba, Hyperci herba, and Plantaginis herba in order to enhance the biological activity. These may interfere in the analysis of the compounds in conventional chromatographic analysis. However, there was no problem in the analysis using the present method. Table 2 shows the results of the quantitation of compounds in the various preparations. The results obtained by the ${ }^{1} \mathrm{H}-\mathrm{NMR}$ method were found to be highly accurate with a standard deviation of $5 \%$ in most cases. The contents of Ginkgo terpenes observed in commercial products were about 4 times higher than the plant material except for sample 2, which contained additional plant materials as mentioned above. The contents of the Ginkgo bioactive compounds could be analyzed with a simple method and in short time, only $5 \mathrm{~min}$, i.e. much shorter than conventional chromatographic methods. Therefore, this ${ }^{1} \mathrm{H}-$
Table 1. Recovery of Bilobalide (BB), Ginkgolide A (GA), Ginkgolide (GB), and Ginkgolide (GC) from Cellulose Paper by $70 \% \mathrm{MeOH}$ Extraction and the Fractionation of Cyclohexane and Methylethylketone Followed by ${ }^{1}$ H-NMR Method $(\% \pm \text { S.D. })^{a)}$

\begin{tabular}{cccc}
\hline \hline BB & GA & GB & GC \\
\hline $105.0( \pm 2.0)$ & $105.3( \pm 4.0)$ & $107.2( \pm 1.2)$ & $96.9( \pm 2.2)$ \\
\hline
\end{tabular}

a) All experiments were based on triplicate measurement.

Table 2. The Contents of Bilobalide (BB), Ginkgolide A (GA), Ginkgolide B (GB), Ginkgolide C (GC) Quantitized by ${ }^{1} \mathrm{H}-\mathrm{NMR}$ Spectrum of the Extracts $\left.(\mathrm{mg} / \mathrm{g} \pm \text { S.D. })^{a}\right)$

\begin{tabular}{lcccc}
\hline \hline Sample & BB & GA & GB & GC \\
\hline Leaves & $1.04( \pm 0.27)$ & $0.75( \pm 0.10)$ & $0.73( \pm 0.05)$ & $0.58( \pm 0.02)$ \\
Sample 1 & $4.85( \pm 0.42)$ & $4.86( \pm 0.36)$ & $3.11( \pm 0.14)$ & $2.64( \pm 0.34)$ \\
Sample 2 & $0.28( \pm 0.03)$ & $0.25( \pm 0.03)$ & $0.18( \pm 0.04)$ & $0.14( \pm 0.06)$ \\
Sample 3 & $4.07( \pm 0.19)$ & $5.74( \pm 0.24)$ & $2.67( \pm 0.20)$ & $3.13( \pm 0.15)$ \\
Sample 4 & $1.38( \pm 0.12)$ & $1.03( \pm 0.09)$ & $0.70( \pm 0.09)$ & $0.75( \pm 0.05)$ \\
Sample 5 & $5.24( \pm 0.31)$ & $3.72( \pm 0.23)$ & $1.83( \pm 0.14)$ & $2.08( \pm 0.12)$ \\
Sample 6 & $3.33( \pm 0.59)$ & $1.40( \pm 0.24)$ & $0.88( \pm 0.17)$ & $1.14( \pm 0.16)$
\end{tabular}

a) All experiments were based on triplicate measurement.

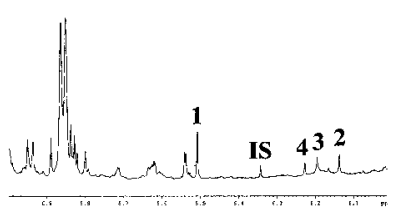

A
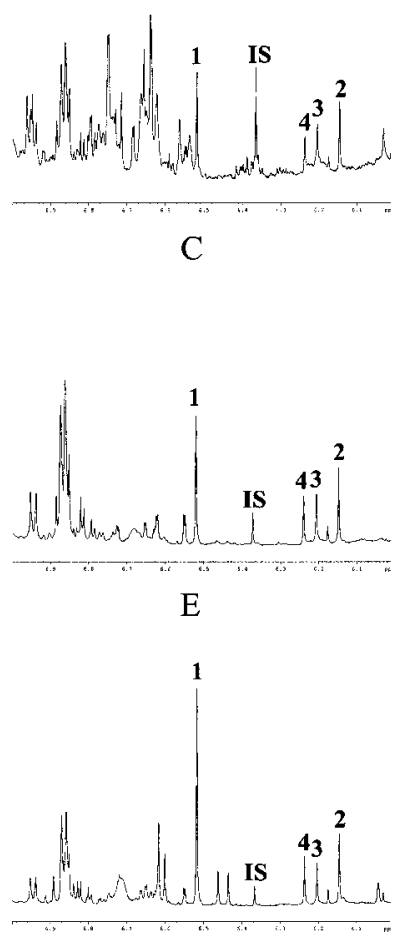

$\mathrm{G}$
$\mathrm{C}$

E

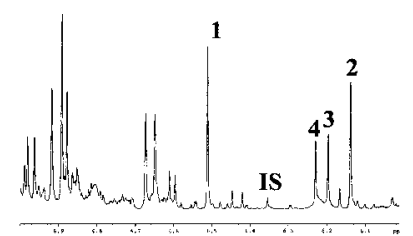

B

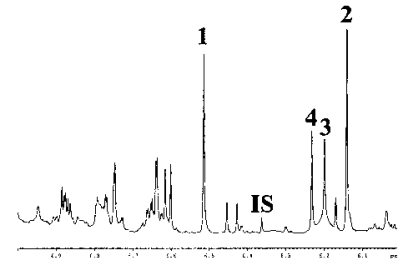

$\mathrm{D}$

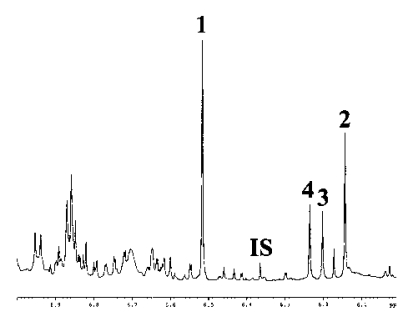

$\mathrm{F}$
Fig. 5. ${ }^{1} \mathrm{H}-\mathrm{NMR}$ Spectra of Ginkgo biloba Leaves (A), Sample 1 (B), 2 (C), 3 (D), 4 (E), 5 (F), and $6(\mathrm{G})$ in the Range of $\delta 6.0-7.0$

1: H-12 of bilobalide (BB), 2: H-12 of ginkgolide A (GA), 3: H-12 of ginkgolide B (GB), 4: H-12 of ginkgolide C (GC), IS: internal standard (phloroglucinol). 
NMR method for the analysis fulfils all the requirements of the commercial Ginkgo products. The ${ }^{1} \mathrm{H}-\mathrm{NMR}$ method has the additional advantage that at the same time it is rapid, simple, and selective and an overall profile is obtained of the preparation. For the pharmaceutical preparation, this profile should be reproducible.

Acknowledgments The Post-doctoral Fellowship Program of Korea Science \& Engineering Foundation (KOSEF) and the Fellowship Program of Universidad Complutense de Madrid/Empresa Flores Valles (Spain) are gratefully acknowledged for their support of Y. H. Choi, H.-K. Choi, and P. Bermejo.

\section{References}

1) Van Beek T. A., "Ginkgo biloba," Chap. 1, ed. by Van Beek T. A., Harwood Academic Publishers, Amsterdam, 2000.

2) Direu K., De Feudis F. V., "Ginkgo biloba," Chap. 17, ed. by Van Beek T. A., Harwood Academic Publishers, Amsterdam, 2000.

3) Chatterjee S. S., "Effects of Ginkgo biloba Extract on Organic Cerebral Impairment," ed. by Agnoli A., Rapin J. R., Scapagnini V., Weitbrecht W. V., Libbey, London, 1984, pp. 5-15.

4) Clostre F., Presse Med., 15, 1529-1538 (1985)

5) Karcher L., Zagermann P., Krieglstein J., Naunyn-Schmiedebergs Arch. Pharmacol., 27, 31-35 (1984).

6) Tang W., Eisenbrand G., "Chinese Drugs of Plant Origin," Springer Verlag, Berlin, 1992.
7) Hasler A., "Ginkgo biloba," Chap. 7, ed. by Van Beek T. A., Harwood Academic Publishers, Amsterdam, 2000.

8) Braquet P., Adv. Prostanglandin Thromboxane Leukotriene Res., 26, 179-198 (1986).

9) Kleijnen J., Knipachild P. J., Brit. J. Clin. Pharmacol., 34, 352-358 (1992).

10) Kleijnen J., Knipachild P. J., Lancet, 340, 1136-1139 (1992).

11) Le Bars P., Katz M. M., Berman N., Itil T. M., Freedman A. M., Schatzberg A. F., JAMA, 278, 1327-1331 (1997).

12) Maurer K., Dierks R. I., Frolich L., J. Psychiat. Res., 31, 645-655 (1997).

13) Pietta P. G., Mauri P. L., Rava A., Chromatographia, 29, 251-253 (1990).

14) Pietta P. G., Mauri P. L., Rava A., J. Pharm. Biomed. Anal., 10, 1077 1079 (1992).

15) Van Beek T. A., Scheeren H. A., Rantio T., Melger W. C., Leyveld G. P., J. Chromatogr., 543, 375-387 (1991).

16) Chen P., Su X.-L., Nie L.-H., Yao S.-Z., J. Chromatogr. Sci., 36, $197-$ 200 (1998).

17) Huh H., Staba E. J., Planta Med., 59, 232-239 (1993).

18) Van Beek T. A., Van Veldhuizen A., Lelyveld G. P., Piron I., Lankhorst P. P., Phytochem. Anal., 4, 262-268 (1993).

19) Markham K. R., "Techniquies of Flavonoid Identification," Academic Press, London, 1982.

20) Komoda Y., Nakamura H., Uchida M., Reports of the Institute for Medicinal \& Dental Egineering, 2, 83-85 (1988). 